\title{
A new approach to simulate the dynamic response of high-tensile chain-link drapery systems
}

\author{
S Tahmasbi University of Newcastle, Australia \\ A Giacomini University of Newcastle, Australia \\ R Bucher Geobrugg Australia Pty Ltd, Australia \\ O Buzzi University of Newcastle, Australia
}

\begin{abstract}
Rockfall draperies are gaining more popularity due to the ease of installation and reduced need for maintenance. Such systems have been around for more than 50 years but limited research has been conducted on their performance and dynamic response. Physical testing of drapery systems typically incurs very high cost and significant technical constraints, all of which can be reduced by resorting to numerical simulations. However, intricate modelling techniques required to capture the realistic response of the systems, leads to computationally expensive numerical models. This paper utilised the previously-developed modelling technique, called hybrid method, to present a computationally efficient numerical model of a chain-link drapery. Commercially available finite element package, ABAQUS/Explicit, was used in this study. The part of the mesh that is in contact with the block and potentially undergoes plasticity and damage was simulated using the real architecture of the wires, while the part of the mesh that remains elastic was simulated using homogenised shell surfaces. Preliminary results showed that the hybrid technique reduces the computational cost of the model to the great extent by reducing the number of degree of freedom as well as minimising the contact zones in the model. The results of the hybrid model were validated against the results of a baseline model, which was fully modelled using the real architecture of the wires. The computational efficiency of the model is also discussed. The developed hybrid model provides an efficient tool to study the dynamic response of drapery systems under different loading conditions.
\end{abstract}

Keywords: ABAQUS, numerical modelling, hybrid method, chain-link, rockfall drapery

\section{Introduction}

Rockfall protection structures are typically categorised as active and passive systems. Active measures directly act on the rock surface preventing instability from occurring, while passive methods are designed to minimise the effects of the falling rocks by intercepting them during their fall and protect the areas at risk. Rockfall draperies are generally listed within the rockfall passive protection methods as they directly control the block trajectory. They are used as low-maintenance (Badger et al. 2008; Cerro et al. 2016; Glover et al. 2012) and effective protection methods in both civil and mining applications. In Australia, drapery systems in combination with rockbolting and concrete portal structures are significantly used to protect punch longwall entry.

Several studies have been conducted on the design of rockfall drapery systems (Bertolo et al. 2009; Giacomini et al. 2012; Muhunthan et al. 2005; Sasiharan et al. 2006; Thoeni et al. 2014). However, given the complex nature of the problem and more importantly its site-specific characteristics, more research is required to better investigate the performance of such systems under various loading conditions. In recent decades, with significant advances in computational technology and due to the large costs and potential risks associated with field experimentation, numerical simulations have become a cost-effective way to study the performance of rockfall protection systems. Sasiharan et al. (2006) reported the use of finite element method to simulate chain-link drapery systems. The authors developed a simplified finite element model simulating 
chain-link drapery systems. The model was developed based on several simplifying assumptions such as ignoring the real architecture of the chain-link wires and considering simplified linear elastic constitutive behaviour for the wires. Although such a simplified modelling technique leads to a huge computational efficiency, many aspects of the complex mesh behaviour cannot be captured, such as the effect of the frictional sliding on the energy dissipation of the system, and most importantly, the wire failure cannot be traced, leading to unrealistic failure patterns of the system.

The focus of the current study is to simulate chain-link drapery systems using the new hybrid approach proposed by Tahmasbi et al. (2019). The new approach is based on identifying two types of mesh zones: the impact zone which undergoes plasticity and damage and peripheral zones which remain elastic throughout the impact. In the hybrid approach, the real architecture of the wires is modelled in the impact zone while the peripheral zones are modelled using homogenised shell surfaces. Such a modelling technique allows capturing the realistic response of the mesh in the impact zone while reducing the computational costs of the model considerably. Accounting for the mass of the peripheral zone is critical to capture the inertia of the system. The hybrid model, developed using commercial finite element package ABAQUS/Explicit 6.14, was used to reproduce the laboratory-scale tests on chain-link drapery systems (Tahmasbi et al. 2018). The validated model was used to simulate a large-scale drapery system for which the computational efficiency and the results accuracy are compared to those of the model entirely simulated using the real architecture of the wires (baseline model).

\section{Hybrid finite element model development}

Three-dimensional geometry of the chain-link mesh with aperture diameter of $\omega=65 \mathrm{~mm}$ and wire diameter of $d=4 \mathrm{~mm}$ (TECCO G65/4) were modelled in ABAQUS (Figure 1). The chain-link wires were used to develop a hybrid finite element (FE) model of the chain-link drapery. The developed hybrid model was first validated against laboratory-scale tests and then used to simulate the large-scale chain-link drapery system.

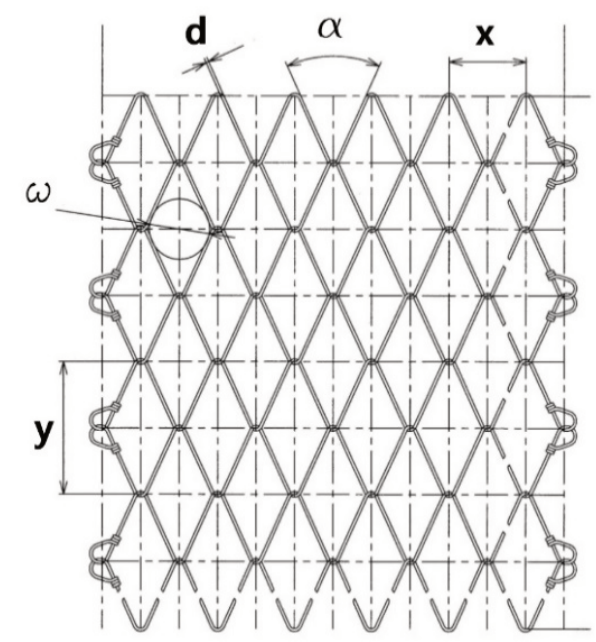

(a)

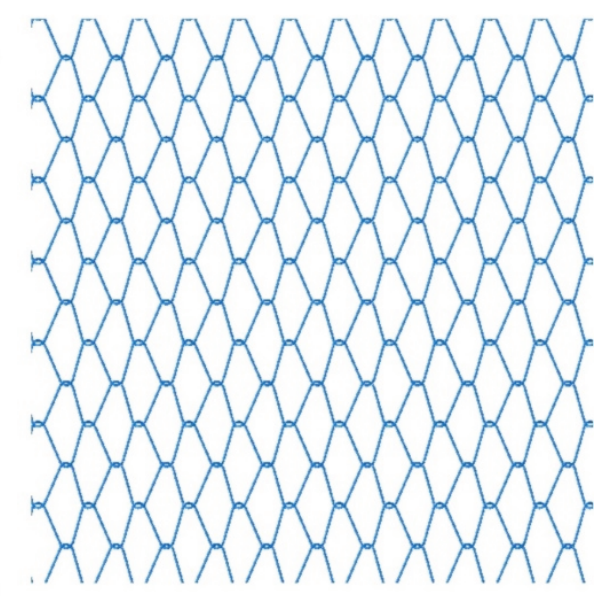

(b)

Figure 1 (a) Geometry of chain-link wires (modified after Geobrugg 2018); (b) Simulated chain-link wires using ABAQUS

\subsection{Hybrid model of laboratory-scale chain-link drapery}

\subsubsection{Model geometry}

The chain-link wires were used to reproduce the results of the laboratory-scale tests reported by Tahmasbi et al. (2018). The test included a free fall of a concrete block released from a specific height to move between the chain-link drapery of size $3.5 \times 3 \mathrm{~m}$ and a concrete slab of size $1.2 \times 3 \mathrm{~m}$. Figure 2 shows the test setup. 


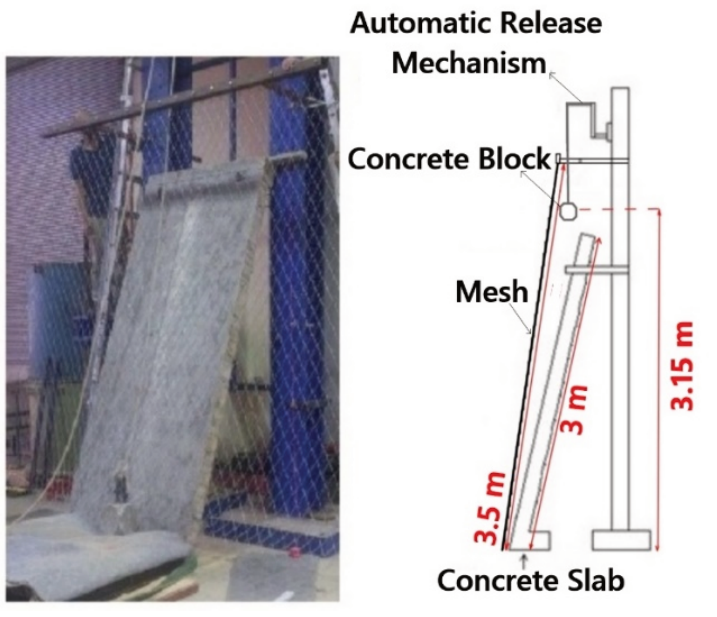

(a)

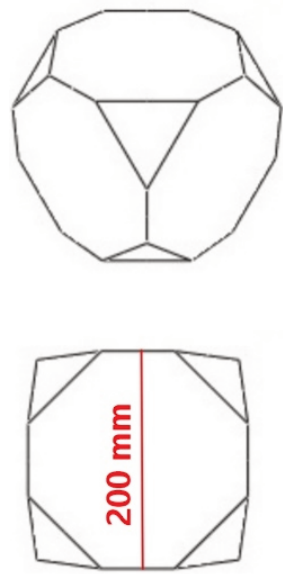

(b)

Figure 2 Laboratory-scale drapery test: (a) Experimental setup; (b) 3D and side views of the concrete block (modified after Tahmasbi et al. 2018)

To develop a hybrid model, the first step is to define the area of the mesh that undergoes plasticisation. To this aim, a FE baseline model of chain-link drapery was used to determine the area of the mesh that undergoes plastic deformation. The result shows that plastic deformation was developed only in a limited area of the mesh, which is in contact with the block. Such observation was also made during the laboratory-scale testing of the drapery mesh. Given the distribution of the plastic strain in the mesh and considering the possible displacement of the block along $x$-axis during the fall, the hybrid model was developed with a central zone of width $1.5 \mathrm{~m}$ and two peripheral zones of width $1 \mathrm{~m}$. It should be noted that the size of the block should be considered in defining the size of the central zone. A total of 36,916 2-node 3D linear beam elements were used to discretise the central zone of the chain-link drapery. Non-uniform discretisation was used in peripheral zones where the size of elements increases from the side to the centre of the peripheral zones. It minimises the effect of stress concentration at shell/beam boundaries allowing capturing the realistic stress gradient in the system. The total number of 60,450 shell elements, with size ranging from $5 \times 5 \mathrm{~mm}$ to $36 \times 73 \mathrm{~mm}$, was used to discretise the peripheral zones. Figure 3 presents the geometry of the hybrid model. Blocks and rock slope were modelled as rigid bodies.

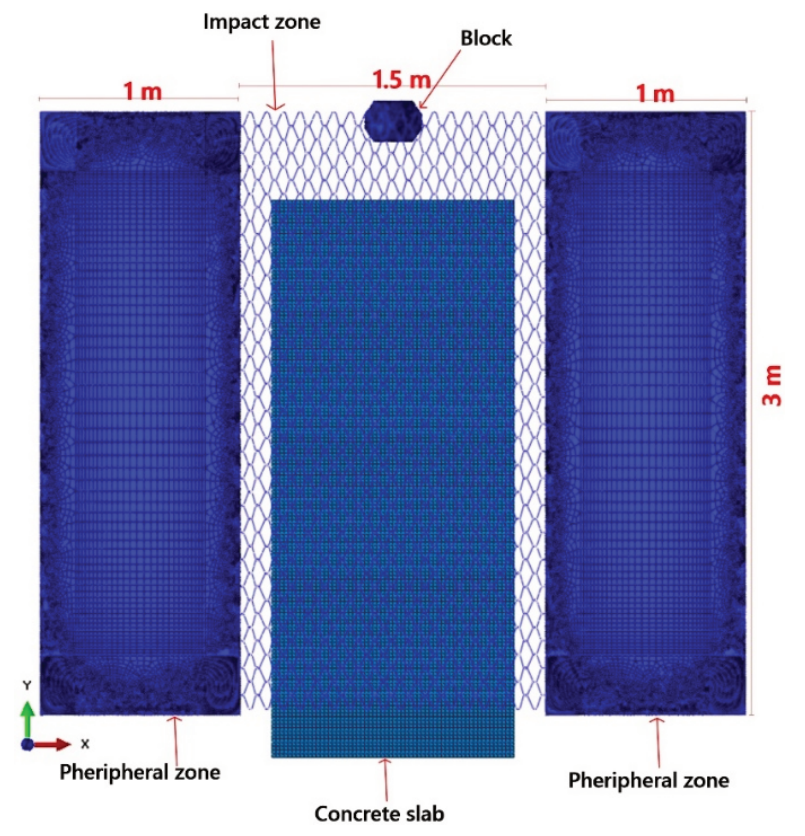

Figure 3 Geometry of the hybrid model 


\subsubsection{Constitutive model}

The hybrid model includes beam elements in the central zone, which undergoes plasticity and possibly damage and shell elements in the peripheral zones, which remain elastic during impact. The constitutive model considered for the beam elements includes elasticity, strain-hardening plasticity and progressive damage. The plastic response of the steel is defined by an isotropic hardening metal plasticity model. The Mises yield surface was introduced in the model by defining uniaxial yield stress $\left(\sigma_{1}, \sigma_{2}, \sigma_{3}\right)$ as a function of plastic strain $\left(\bar{\varepsilon}_{1}^{p l}, \bar{\varepsilon}_{2}^{p l}, \bar{\varepsilon}_{3}^{p l}\right)$. Steel wire failure is achieved through ductile damage initiation criterion in combination with an exponential damage evolution law, requiring the definition of the plastic strain at the onset of damage $\left(\bar{\varepsilon}_{d}^{p l}\right)$, the equivalent plastic displacement at failure $\left(\bar{u}_{f}^{p l}\right)$ and the exponent $\left(\alpha_{\text {damage }}\right)$ that defines the exponential relation between the damage factor and the equivalent plastic strain. The constitutive behaviour of the shell elements is modelled using orthotropic elasticity requiring nine independent parameters to be defined. The constitutive behaviour of beam and shell elements were calibrated against experimental drop tests in terms of block trajectory and velocity throughout the impact (Tahmasbi et al. 2019) and carried over for this study. Tables 1 and 2 summarise the constitutive parameters for the beam and shell respectively. The reader is invited to consult the publications by Tahmasbi et al. (2019) for more details.

Table 1 Material constitutive parameters for beam elements

\begin{tabular}{ll}
\hline Material parameter & Input value \\
\hline Young's modulus (EOTA) & 210,000 \\
Poisson's ratio & 0.3 \\
{$\left[\sigma_{1}(\mathrm{MPa}), \varepsilon_{1}^{p l}\right]$} & {$[1,770,0]$} \\
{$\left[\sigma_{2}(\mathrm{MPa}), \varepsilon_{2}^{p l}\right]$} & {$[2,000,0.015]$} \\
{$\left[\sigma_{3}(\mathrm{MPa}), \varepsilon_{3}^{p l}\right]$} & {$[2,250,0.15]$} \\
Fracture strain $\left(\bar{\varepsilon}_{d}^{p l}\right)$ & 0.08 \\
Exponent $\left(\alpha_{\text {damage }}\right)$ & 1 \\
Equivalent plastic displacement at failure $\left(\bar{u}_{f}^{p l}\right)(\mathrm{mm})$ & 1.8 \\
\hline
\end{tabular}

Table 2 Material constitutive parameters for shell elements

\begin{tabular}{llll}
\hline Material parameter & Input value & Material parameter & Input value \\
\hline$E_{x}(\mathrm{MPa})$ & 700 & $v_{y z}$ & 0.3 \\
$E_{y}(\mathrm{MPa})$ & 400 & $G_{x y}(\mathrm{MPa})$ & 26.25 \\
$E_{z}(\mathrm{MPa})$ & 200 & $G_{x z}(\mathrm{MPa})$ & 52.5 \\
$v_{x y}$ & 0.3 & $G_{y z}(\mathrm{MPa})$ & 75 \\
$v_{x z}$ & 0.3 & - & - \\
\hline
\end{tabular}

\subsection{Hybrid model of large-scale chain-link drapery}

Once the laboratory-scale hybrid model is validated against experimental data (results are presented in section 4.1), it was used to develop a hybrid model of the large-scale chain-link drapery system. The model includes a drapery mesh of size $8 \times 16 \mathrm{~m}$ (width $\times$ height), a block of size $860 \mathrm{~mm}$ and a rock slope of size $10 \times 19 \mathrm{~m}$ (width $\times$ height). The block geometry is based on European Organisation of Technical Approvals [EOTA] 2008. 
The preliminary simulations using the baseline model of the same size shows that only the central part of the mesh, which is in contact with the block, undergoes plastic strain (Figure 4a). Accordingly, the hybrid model with one central zone of width $2 \mathrm{~m}$ and two peripheral zones of width $3 \mathrm{~m}$ was developed. Figure 4 shows the distribution of plastic strain within the baseline model and the geometry of the hybrid mesh.

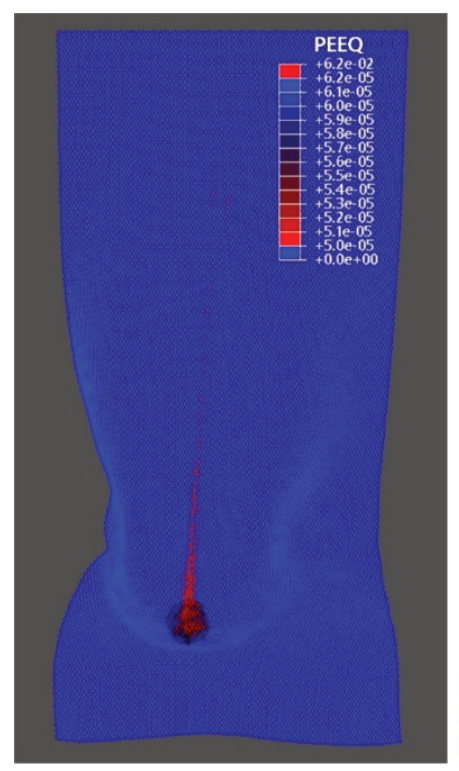

(a)

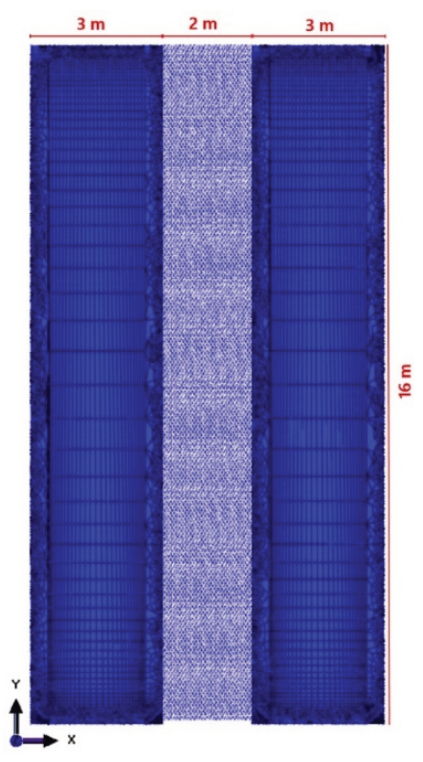

(b)

Figure 4 (a) Distribution of plastic strain in the large-scale baseline model; (b) Geometry of the hybrid mesh

The geometry of the rock slope considered in this study is according to the 3D model of the highwalls presented in Thoeni et al. (2014). Accounting for a rock slope with random surface roughness, rather than a smooth surface used in Section 2.1.1, allows more realistic simulation of the mesh/rock slope interaction. The rock slope was discretised using total number of 241,308 rigid elements of type 3-node triangular facet (R3D3) and 4-node bilinear quadrilateral (R3D4) elements. The rock slope, the mesh and the block were assembled to develop a model of a drapery system. The mesh was positioned near the rock slope far enough for the block to be positioned between them. Three anchors were considered at the top line of the mesh. They were modelled as fixities and located with the same distance from each other. Figure 5 shows the assembly of the hybrid model.

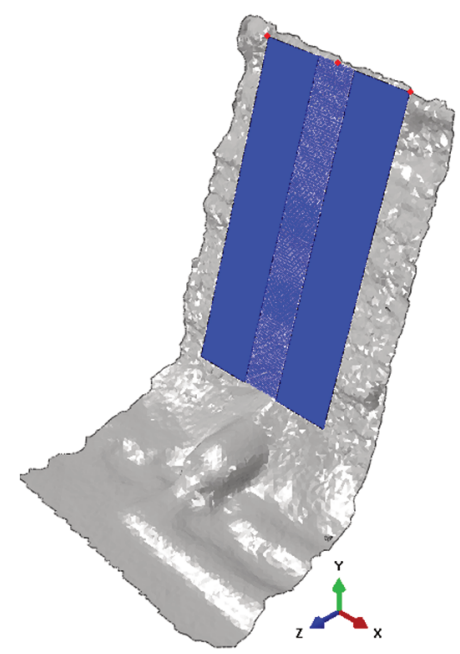

(a)

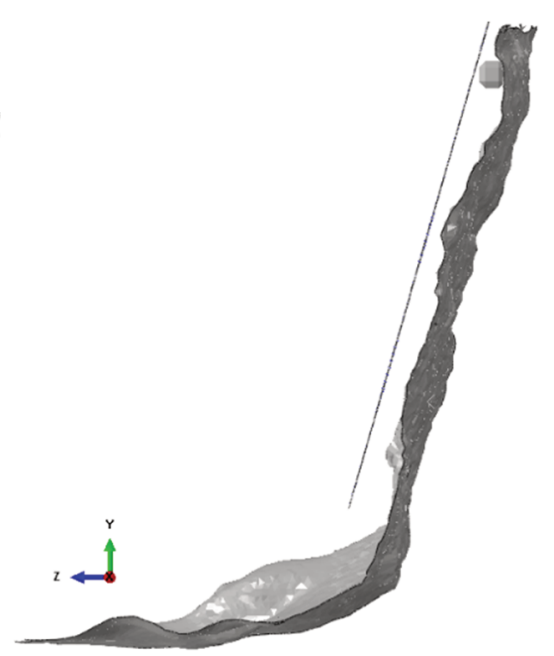

(b)

Figure 5 (a) 3D view of hybrid model assembly; (b) Side view of hybrid model assembly. The red dots show the location of the anchors 


\subsection{Simulation procedure}

The baseline and hybrid models of laboratory-scale and large-scale drapery systems were simulated following the same simulation procedure. The analyses were done in two steps: the gravity step and the impact step. During the gravity step, the mesh was loaded with gravitational force in order to engage the wires and place the mesh on the slope. The block, which was fixed during the gravity step, was released from its initial position to free fall under gravity and move between the mesh and the rock slope. Mass-scaling technique was used to reduce the computational costs of the models.

\section{Results and discussion}

This section presents the results obtained from hybrid models in comparison to those from baseline models. The accuracy of hybrid models' predictions were compared to those of baseline models in terms of block vertical velocity and block trajectory. The computational efficiency of the hybrid approach was also investigated for both laboratory-scale and large-scale models.

\subsection{Results accuracy of the hybrid models}

Figures $6 a$ and $6 \mathrm{~b}$ show the evolution of block velocity in time and block trajectory in terms of vertical displacement versus horizontal displacement. Point $A$ in Figure 6a refers to the moment when the block impacts the slope and point $B$ refers to the moment at which the block bounces off the slope. As it can be seen from Figure 6 , the hybrid model is capable of reproducing the test results with a reasonable accuracy. The experimental and numerical results start to diverge close to the second impact (point $C$ in Figures $6 a$ and $6 b)$, which is due to lighting conditions during the experiment. A similar comparison was carried out for the large-scale model and the results suggest that the hybrid model is also capable of reproducing the results of the baseline model with reasonable accuracy (Figure 7).

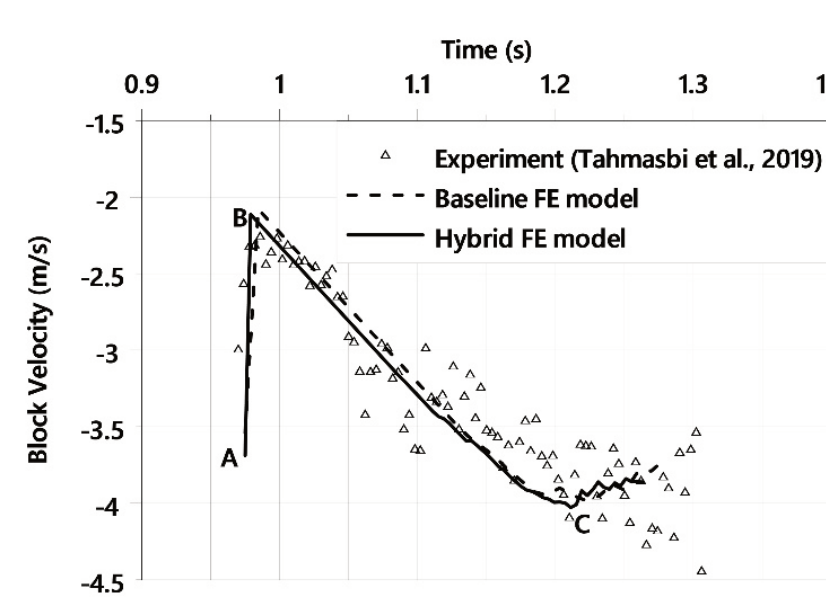

(a)

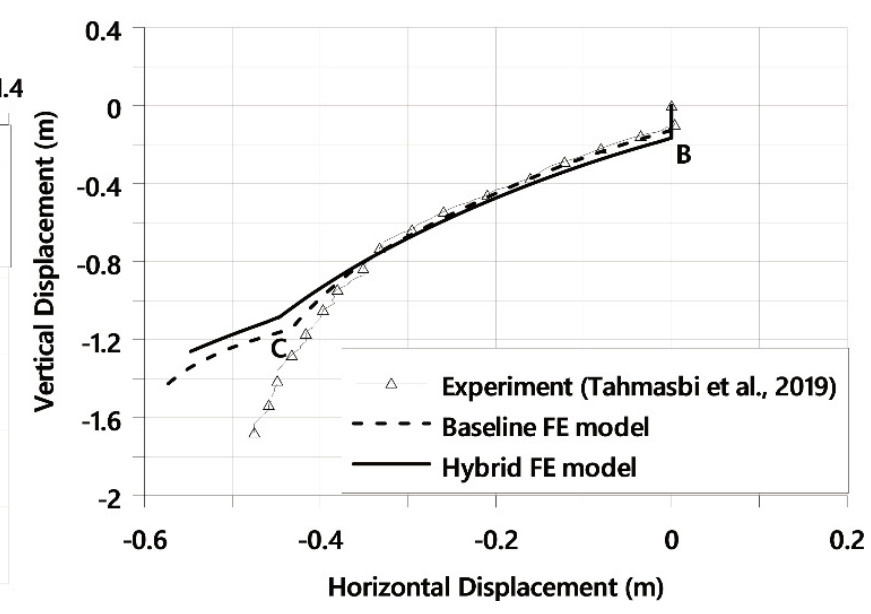

(b)

Figure 6 Comparison of experimental and numerical (baseline and hybrid) results from laboratory-scale tests. (a) Evolutions of block velocity; (b) Evolutions of block trajectory 


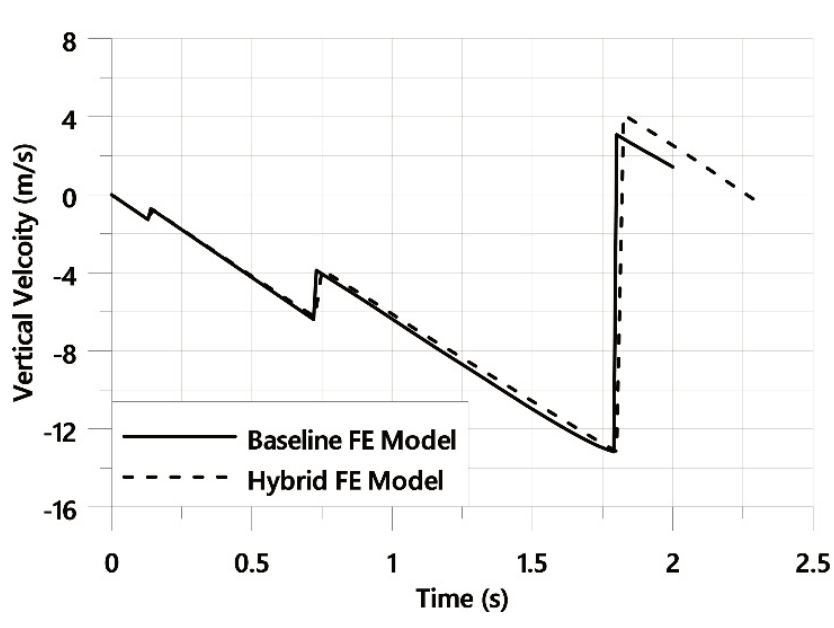

(a)

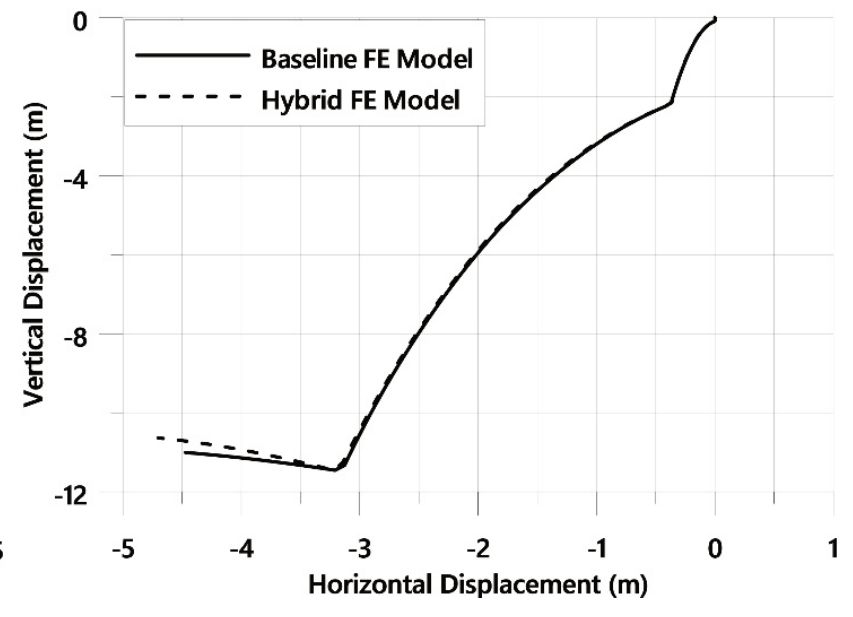

(b)

Figure 7 Comparison of the numerical results from large-scale baseline and hybrid models. (a) Evolutions of block velocity; (b) Evolutions of block trajectory

\subsection{Computational efficiency of the hybrid models}

Here, the computational efficiency of the hybrid models is evaluated against that of the baseline FE models. The laboratory-scale simulations were conducted using a 64-bit Dell OptiPlex 7040 Desktop with four Intel 3.4 GHz processors and $16 \mathrm{~GB}$ of RAM, while an Intel(R) Xeon(R) Gold 6150 server with $345 \mathrm{~GB}$ of RAM and 18 processors of $2.7 \mathrm{GHz}$ frequency were used for large-scale simulations. Figures 8 and 9 compare the computational efficiency for laboratory-scale and large-scale models respectively in terms of CPU time, run time and memory utilisation. Run time and CPU time are normalised against the run time and CPU time of the baseline model. Run time is calculated from the onset of analysis to its end, while CPU time excludes the time required for data input/output. The results for the laboratory-scale model show that replacing $57 \%$ of the mesh area with shell surfaces leads to almost $26 \%$ reduction in CPU time and run time. The baseline model utilises $9.3 \%$ of the available memory while $8.4 \%$ was utilised by the hybrid model. Regarding the large-scale model, replacing $75 \%$ of the mesh area with shell surfaces resulted in almost $57 \%$ saving in CPU time and run time. Only $5 \%$ of the available memory was utilised by the large-scale hybrid model, while more than $17 \%$ of the available memory was occupied when running baseline model. Reduction in the computational costs of the model can be attributed to less contact zones in the hybrid models compared to the baseline models. Higher computational efficiency of the hybrid approach for the large-scale model compared to the laboratory-scale model can be explained by larger area of the mesh replaced with shell surfaces as well as the possibility of using larger shell elements at the centre of peripheral zones far from the boundaries for the large-scale model. 


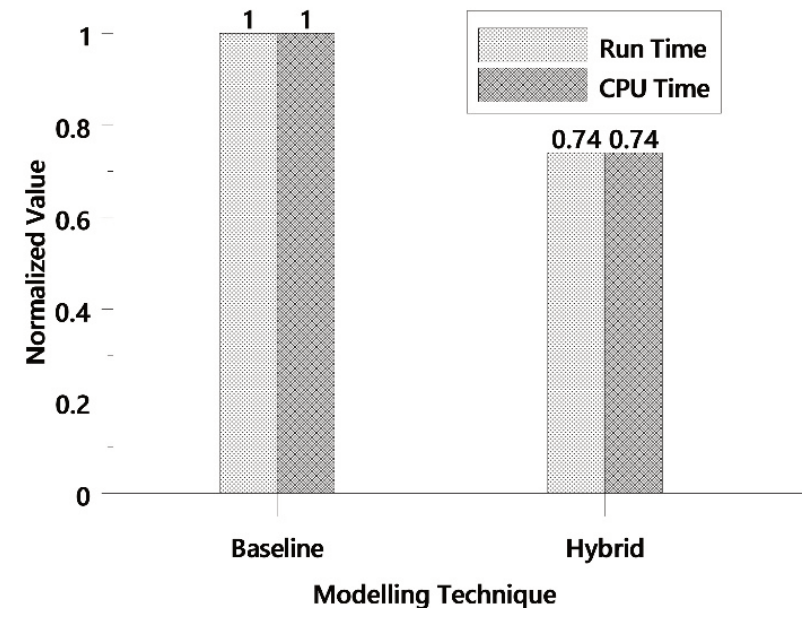

(a)

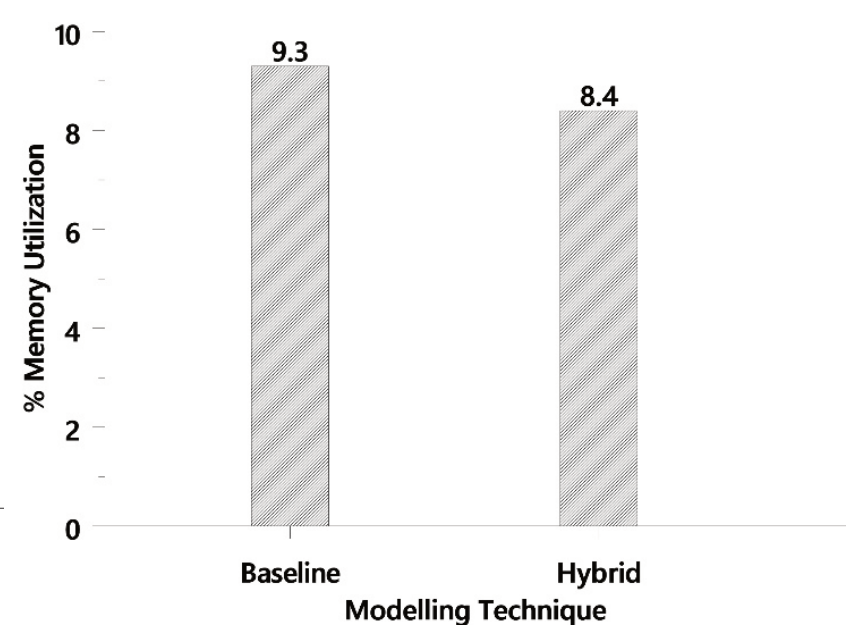

(b)

Figure 8 Computational cost of baseline and hybrid models of laboratory-scale drapery. (a) Run time and CPU time; (b) Percentage of memory utilisation

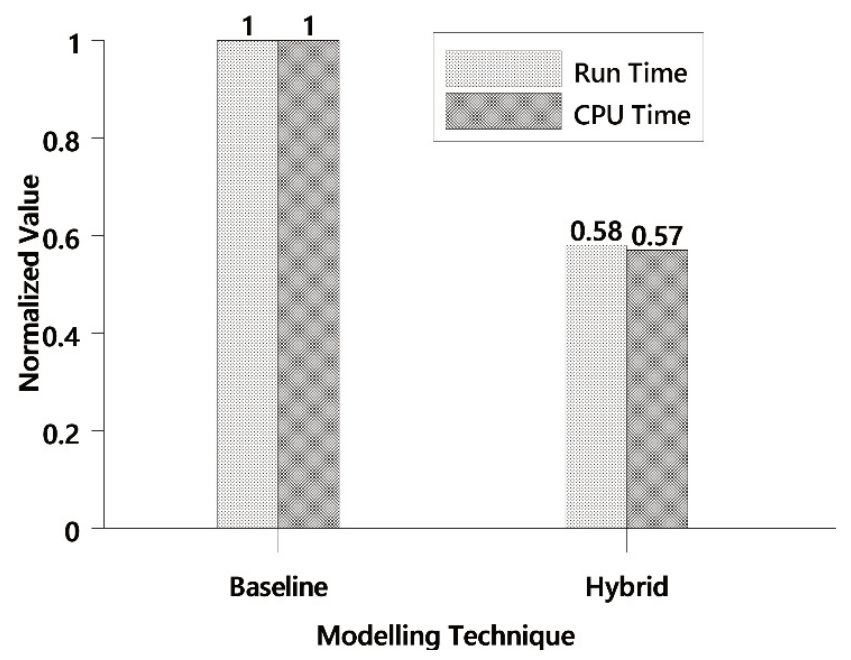

(a)

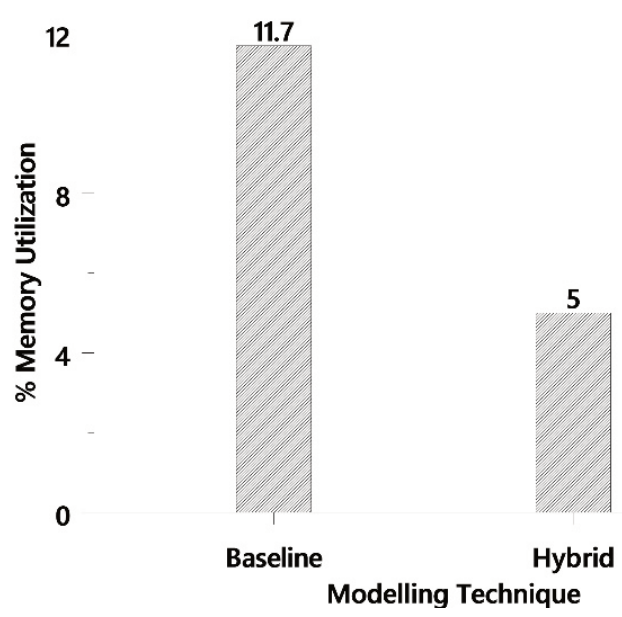

(b)

Figure 9 Computational cost of baseline and hybrid models of large-scale drapery. (a) Run time and CPU time; (b) Percentage of memory utilisation

\section{Conclusion}

A computationally efficient approach called hybrid approach was utilised to simulate chain-link drapery system using ABAQUS FE code. To this aim, two different zones were identified within the drapery mesh based on the mesh deformation: the central zone, which is in contact with the block and undergoes plasticity and damage and the peripheral zones, which remain mostly elastic during the impact. The hybrid approach is based on accounting for the real architecture of the wires in the central zone, while using homogenised shell surfaces in the peripheral zones. The model was used to reproduce the results of the laboratory-scale tests on chain-link drapery. The prediction capability of the model was validated in terms of block velocity and block trajectory. The validated model was used to develop a large-scale model of the chain-link drapery system. The accuracy of the results from large-scale hybrid model was verified against those from the baseline model. The results show that the hybrid approach reduces the computational costs of the model to a considerable extent both in terms of run time and memory utilisation, while its effect on the results accuracy is negligible. The validated large-scale hybrid model will be used to conduct parametric studies on the design parameters of the chain-link drapery systems. 


\section{Acknowledgement}

The authors would like to acknowledge the financial and in-kind support received by Geobrugg AG for this research.

\section{References}

Badger, T, Duffy, J, Sassudelli, F, Ingraham, PC, Perreault, P, Muhunthan, B,...\& Castelli, E 2008, 'Hybrid Barrier Systems for Rockfall Protection', in A Volkwein, V Labiouse \& K Schellenberg (eds), Proceedings of the Interdisciplinary Workshop on Rockfall Protection, Eidg. Forschungsanstalt WSL, Birmensdorf, pp. 10-12.

Bertolo, P, Oggeri, C \& Peilab, D 2009, 'Full-scale testing of draped nets for rock fall protection', Canadian Geotechnical Journal, vol. 46, issue 3, pp. 306-317.

Cerro, M, Giacchetti, G, Lelli, M, Grimod, A \& Arul, A 2016, 'Hybrid rockfall barrier - new design methodology based on the Colorado full-scale test experience', in PM Dight (ed.), Proceedings of the First Asia Pacific Slope Stability in Mining Conference, Australian Centre for Geomechanics, Perth, pp 393-406.

European Organisation of Technical Approvals 2008, ETAG 027: Guideline for European Technical Approval of Falling Rock Protection Kits, https://www.empa.ch/documents/20659/66481/Dienstleistungen_Bewertungsstelle_ETAG-027.pdf/f4d9d2c0-c2ce4cc4-b307-3d751cdd6f18

Giacomini, A, Thoeni, K, Lambert, C, Booth, S \& Sloan, SW 2012, 'Experimental study on rockfall drapery systems for open pit highwalls', International Journal of Rock Mechanics and Mining Sciences, vol. 56, pp. 171-181.

Geobrugg 2018, High-tensile steel wire mesh TECCO G65/4, Geobrugg, https://www.geobrugg.com/ datei.php?src=portal/downloadcenter/dateien/downloadcenter/level1-data-sheets/TECCO/L1_TECCO-G65-4mm_TechData _190506_e.-20m.pdf

Glover, J, Denk, M, Bourrier, F, Volkwein, A \& Gerber, W 2012, 'Measuring the kinetic energy dissipation effects of rock fall attenuating systems with video analysis', Proceedings of the12th Congress INTERPRAEVENT, International Research Society INTERPRAEVENT, Klagenfurt, pp. 151-160.

Muhunthan, B, Shu, S, Sasiharan, N \& Hattamleh, OA 2005, Analysis and design of wire mesh/cable net slope protection, Washington State Transport Commission, Washington, https://www.wsdot.wa.gov/research/reports/fullreports/612.1.pdf

Sasiharan, N, Muhunthan, B, Badger, TC, Shu, S \& Carradine, DM 2006, 'Numerical analysis of the performance of wire mesh and cable net rockfall protection systems', Engineering Geology, vol. 88, issue 1-2, pp. 121-132.

Tahmasbi, S, Giacomini, A, Wendeler, C \& Buzzi, O 2018, '3D finite element modelling of chain-link drapery system', in V Litvinenko (ed.), Proceedings of the European Rock Mechanics Symposium, Taylor and Francis Group, Saint Petersburg.

Tahmasbi, S, Giacomini, A, Wendeler, C \& Buzzi, O 2019, 'On the Computational Efficiency of the Hybrid Approach in Numerical Simulation of Rockall Flexible Chain-Link Mesh', Rock Mechanics and Rock Engineering, vol. 52, issue 10, pp. 3849-3866.

Thoeni, K, Giacomini, A, Lambert, C, Sloan, SW \& Carter, JP 2014, 'A 3D discrete element modelling approach for rockfall analysis with drapery systems', International Journal of Rock Mechanics \& Mining Sciences, vol. 68, pp. 107-109. 
